ISSN 2073-8994

www.mdpi.com/journal/symmetry

Article

\title{
The Relationship Between Morphological Symmetry and Immune Response in Wild-Caught Adult Bush-Crickets
}

\author{
Åsa Berggren* and Matthew Low \\ Department of Ecology, PO Box 7044, Swedish University of Agricultural Sciences, 75007, Uppsala, \\ Sweden; E-mail: Matt.Low@ekol.slu.se (M.L.) \\ * Author to whom correspondence should be addressed: E-mail: Asa.Berggren@ekol.slu.se.
}

Received: 11 August 2009; in revised form: 17 September 2009 / Accepted: 24 September 2009 / Published: 28 September 2009

\begin{abstract}
Despite interest in the relationship between fluctuating asymmetry (FA), immune response and ecological factors in insects, little data are available from wild populations. In this study we measured FA and immune response in 370 wild-caught male bush-crickets, Metrioptera roeseli, from 20 experimentally introduced populations in southern-central Sweden. Individuals with more-symmetric wings had a higher immune response as measured by the cellular encapsulation of a surgically-implanted nylon monofilament. However, we found no relationship between measures of FA in other organs (i.e. tibia and maxillary palp) and immune response, suggesting that this pattern may reflect differing selection pressures.
\end{abstract}

Keywords: artificial parasite; experiment; fluctuating asymmetry; developmental instability; wings; grasshopper; Roesel’s bush cricket; Orthoptera; connectivity

\section{Introduction}

During development, an organism is exposed to many environmental stressors which may impair optimal growth or physiological functioning [1,2]. Thus, departures from the ideal expression of particular traits are commonly used as evidence that organisms have been exposed to sub-optimal resources as mediated through environmental or genetic constraints [3, 4]. Because immune defence and optimal growth may be traded-off when conditions are less than ideal, the relationship between the expression of these traits and numerous ecological correlates have received increasing attention in evolutionary ecology [5-8]. Fluctuating asymmetry (FA; the deviation of an identical expression of a 
trait across an axis of symmetry) and immune defence (the cellular immune response to exposure to a novel antigen) are often used in such studies as proxies for individual quality [9, 10].

Because of their short generation times and the relative ease at which environmental conditions can be manipulated, insects are particularly well-suited to studying relationships between morphology, immune function and ecological correlates. Although growth and immune response are predicted to be traded-off against each other when conditions are poor [5], direct comparisons of FA and immune function are rare in captive populations (but see [6, 11]) and virtually absent from wild insect populations. Thus for many situations, predicted relationships have not been demonstrated empirically. One reason for the paucity of wild-population studies is that factors to which individuals were exposed during their development and the population's history are usually unknown, which are often the variables of interest in evolutionary ecology studies [9].

In the mid-1990s a large-scale experimental introduction of Roesel's bush-crickets (Metrioptera roeseli, Orthoptera) was initiated in southern-central Sweden. Populations were introduced to 70 locations with varying propagule sizes and landscape variables to study the effects of these factors on population establishment and persistence $[12,13]$. Since then, these populations have been regularly monitored and show that propagule size and habitat connectivity affect population persistence and growth [12, 14], and that patch size and connectivity are correlated with immune response and FA [13, 15]. Because these relationships may be, at least in part, related to genetic diversity in isolated populations [13, 15], the landscape and population history factors which may confound relationships between immune response and FA need to be accounted for. Such information is usually not available in studies of wild populations; thus, our study populations present a rare opportunity to account for these factors when studying relationships between the ecology, FA and immune function in wild insect populations.

In this study we utilise these population histories to control for landscape variables known to influence immune response and FA [13, 15] when comparing the variation in immune response relative to the degree of FA in wild-caught bush-crickets. Individuals that are more symmetrical (lower FA) are generally predicted to have a higher immune response because: (1) poor condition (whether from genetic or environmental factors) should result in larger FAs and higher exposure or susceptibility to parasites [8, 16], or (2) parasites may exploit the limited resources of their hosts during development, which, in turn, increases FA [6]. However it is also conceivable that immune response and FA exhibit a positive relationship because immune defence is traded-off against some other attribute (e.g. symmetry). Thus, we make no predictions as to the direction of any relationships found in this study.

\section{Methods}

\subsection{Study species \& population}

Roesel's bush-cricket is a small species, $12-18 \mathrm{~mm}$ long [17], and is common in south and central Europe, Finland, and Latvia. In Sweden the species is predominantly found in the southeast around Lake Mälaren and is currently undergoing a range expansion [18]. Their preferred habitat is moist ungrazed high-grass areas, where they feed on grass tissue, grass seeds, and small insects [19]. Eggs 
are laid in grass stems during summer and autumn and hatch in May 1 or 2 years later [20]. The nymphs progress through six instars before becoming adults [19]. In Sweden adult males stridulate from July to October, and if the weather is warm or sunny they stridulate almost continuously during the day at this time. The song is characteristic, making the adult males of this species easy to survey.

For this study we collected a total of 377 males from 20 experimentally-introduced populations (mean \pm SE, $18.5 \pm 0.3$ per population, range 16-22). These populations were introduced as a part of a large-scale introduction experiment in 1994 and 1995 (for more details see [12, 13]). At the time of collection, the bush-crickets had been in their adult form for approximately 2 months.

\subsection{The immune-response experiment}

We measured the immune response in each individual by using a standard protocol to provoke the immune system. This was done by experimentally implanting a nylon monofilament under each animal's cuticula, exposed to the circulating haemolyph and positioned along the individual's length (see also [11, 21, 22]. This method effectively measures immune response because when the insect's immune system encounters the monofilament it responds as if the object was biologically important; there is a deposition of melanin precursors and haemocytes encapsulating the object, which darken to form a melanin layer in the capsule [23]. This response can then be measured as the volume of cells attached to the filament and degree of cell melanisation. After implanting the filament, individuals were released back into temperature-controlled $\left(+20^{\circ} \mathrm{C}\right)$ large cages and kept for 24 hours before being euthanased by freezing. After euthanasia, the nylon thread and the cells attached to the thread were carefully dissected out of the abdomen and fixed onto a microscope slide. These were then photographed through a microscope using a digital camera (Nikon, Coolpix 990); the light, exposure and magnification settings were kept constant for all samples. Each photograph was then analysed in a photo-editing software package (Adobe Photoshop Elements Editor; Adobe Systems Incorporated, 2005), where cell aggregation was quantified by counting the number of pixels encompassed by this area (minus the implant) on the digital image. Melanisation was calculated by using the software's histogram function to derive a numerical estimate of brightness [21]; this was then converted to a more-intuitive scale so that increasing values corresponded to increasing levels of melanin (and hence immune function - for more details see [15]).

\subsection{Asymmetry}

For each bush-cricket, a micro-enhancer with built-in ruler $( \pm 0.01 \mathrm{~mm})$ was used to measure three traits: (1) maxillary palp length - distance from the maxillary palp joint to the end of the maxillary palp, (2) fore-wing length - distance from the wing joint to the end of the radial sector and (3) hind tibia length - distance from the femur/tibia joint to the tibia/tarsus joint. FA was measured as the absolute value of the left-right difference [24]. An estimate of measurement error is required when testing for the presence of FA to determine whether between-sides variation is significantly larger than measurement error; we tested for this in a mixed model two-way analysis of variance performed on repeated measures. The analysis of measurement error showed that FA was significant relative to measurement error in all three traits: maxillary palps (MS $=1.53, p<0.05$ ), tibias ( $\mathrm{MS}=0.80, p<$ 0.0001) and wings (MS $=32.81, p<0.0001$; for more details see [13]). An additional composite 
measure of FA was also calculated for each individual as the first component of a principal components analysis (PCA) from the three FA measures.

\subsection{Statistical Analysis}

We used a generalized linear mixed model framework for comparing the relationship between immune response and FA. This allowed us to account for the specific landscape variables which were common to the individuals from each population (i.e. patch size and connectivity) which have been previously shown to affect FA and/or immune response of bush-crickets in this system $[13,15]$ and to include population identity to account for local population effects. In addition, individual body size has also been shown to be correlated with measures of immune function in this species [25]; thus, these factors (population landscape factors and individual body size) were included as random effects in the mixed models. Because cellular immune response can be measured in more than one way, for each FA measure (maxillary palp, wing and tibia) we ran two different models (i.e. where immune defence was defined as either the size of cell aggregation or the degree of melanisation of the capsule). Sample sizes vary between some of the tests because some traits could not be measured for all individuals (e.g. because of a broken or missing hind limb). Means are presented $\pm 1 \mathrm{SE}$. All analyses were carried out using ASReml [26].

\section{Results}

Individuals with higher immune responses, as measured by cell melanisation at the implant site, were more likely to have symmetrical wings (or lower wing FA). There was no significant relationship between immune response and tibia or maxillary palp symmetry (Table 1; Fig. 1); however, there was a trend $(P<0.1)$ for maxillary palps to be more symmetrical in individuals with lower rates of cell deposition at the implant site (Table 1).

Table 1: Results from the generalized mixed model analyses. FA measures were included as fixed effects and population ecological variables and body size included as random effects.

\begin{tabular}{cccc}
\hline \multirow{2}{*}{ FA measure } & NDF, DDF & \multicolumn{2}{c}{ Immune response measure (F, P-value) } \\
\cline { 3 - 4 } & & Cell area & Melanisation \\
\hline Wing & $1,340.4$ & $0.12,0.74$ & $4.5,0.035$ \\
Tibia & $1,225.4$ & $2.22,0.14$ & $0.77,0.38$ \\
Maxillary palp & $1,343.7$ & $3.04,0.083$ & $0.17,0.68$ \\
Composite FA & $1,224.2$ & $0.52,0.61$ & $0.45,0.65$ \\
\hline
\end{tabular}


Figure 1. Relationship between measures of fluctuating asymmetry (absolute left-right difference) and the two immune response measures. The only significant relationship was between degree of melanisation and wing asymmetry (all figures show the uncorrected raw data).
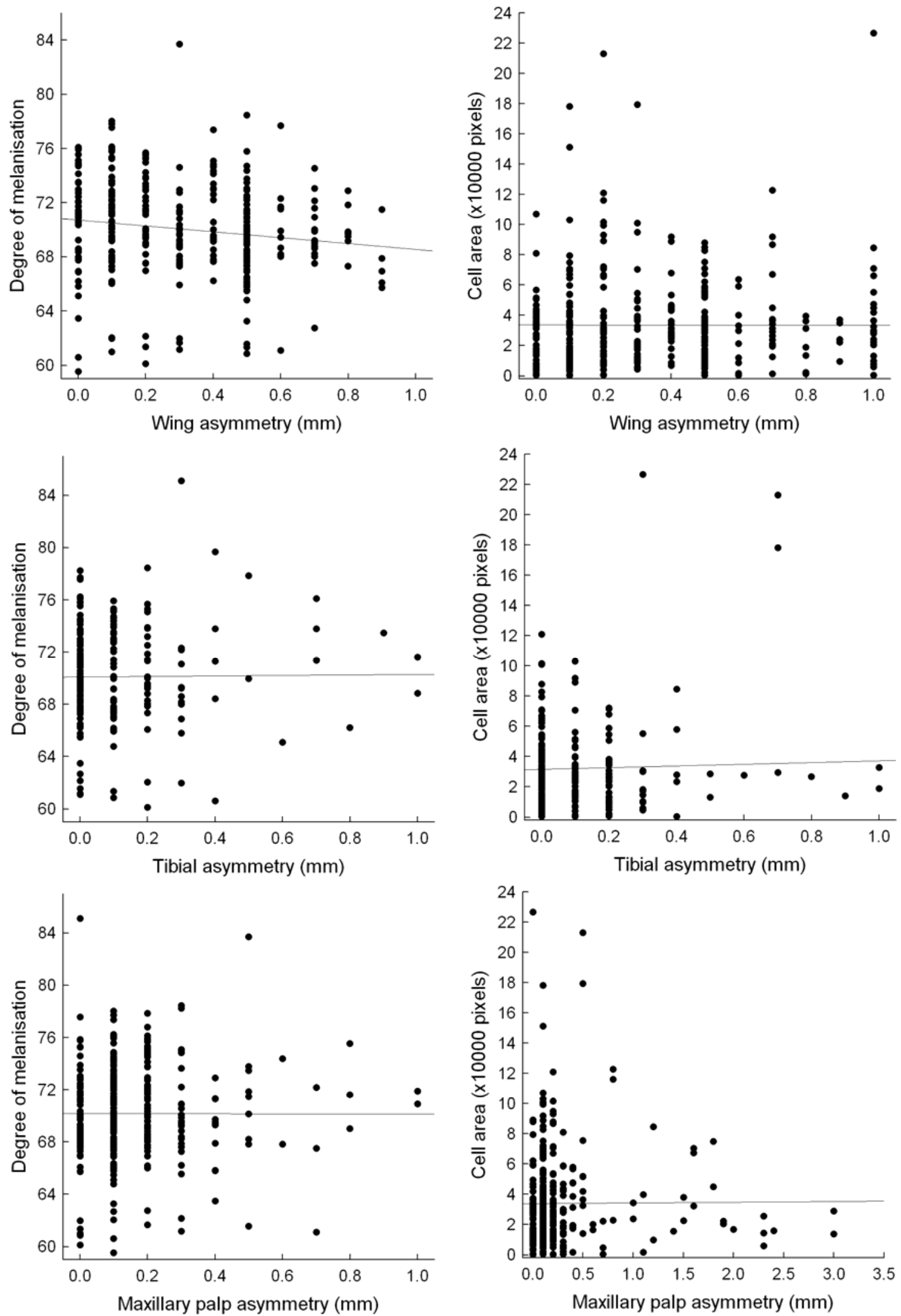


\section{Discussion}

As is predicted by the prevailing literature, individuals with more-symmetrical wings (lower FA) had a higher immune response (melanisation) when presented with a novel antigen. However, significant correlations between immune response and FA were not found for other body parts (i.e. tibia or maxillary palp) or for the composite measure of FA. Below we discuss three explanations for these patterns: (1) there is a correlation between immune response and FA in adult bush-crickets; however, the relationship is restricted to the FA of specific organs (e.g. wings but not legs or mouthparts), (2) there is no correlation between immune response and FA in bush-crickets; i.e. the composite FA-immune response relationship is robust, while the wing FA-immune response relationship is spurious, and (3) correlations generally exist between immune response and FA in bushcrickets; however, these trade-offs occur earlier in development and are not easily detected in mature individuals.

If a relationship between symmetry and immune response exists for wings but not other organs in bush-crickets, then this suggests that sub-optimal individuals (with their sub-optimal status arising from environmental or genetic constraints; $[3,4]$ ) will trade-off optimal growth in wings ahead of that in legs or mouthparts. This would only make sense if wing symmetry was less correlated with fitness than symmetry in other parts of the body. Such a result is plausible when considering that the wings of adult Roesel's bush-crickets play a minor role in the day-to-day survival of individuals - i.e. 99\% of individuals are short-winged and do not fly [27]( $\AA$ Berggren pers. obs.), instead relying on their legs for locomotion within their grass-stem habitat, and their mouthparts for grasping in ingesting food. However, male Roesel's bush-crickets stridulate their wings to attract females and, thus, these organs should be under significant sexual selection [28]. This raises the question of why symmetry is only related to immune function for a sexual ornament (i.e. the stridulation organs - wings) but not for nonornamental traits (i.e. legs and mouthparts) when all traits are expected to be important determinants of fitness (cf. $(6,22])$.

We offer three explanations for why immune function is correlated with developmental instability in wings but not legs or mouthparts in this species. First, FA in wings, at the levels detected in this study, may have no appreciable effect on stridulation quality and, thus, be invisible to selection - while a similar degree of FA in other organs has some effect on survival. If so, it would pay an individual to maintain symmetry in legs and mouthparts, at the expense of wing symmetry, when resources were limited. Second, wings might be more sensitive than other body parts to resource constraints because of reasons unrelated to selection pressures: resulting in covariance in developmental instability and immune response. Such a relationship could be enhanced, rather than diminished by sexual selection, with stridulation being favoured by females as an honest signal of individual quality because of this sensitivity to individual and environmental quality [29]. Finally, legs and mouthparts are probably strongly linked to fitness for both sexes; however, wings are only linked to fitness in males (since females do not stridulate). Thus, selection on the relative resources diverted to different organs in times of stress might be smaller for wings when compared to other organs, because selection is relaxed in females.

Despite the significant correlation between wing FA and immune response found in this study, we recommend some caution in interpreting the robustness of this relationship. Because we examined 
more than one possible relationship between FA and immune response, it could be argued that we need to account for this by modifying the significance level via some sort of Bonferroni correction (e.g. [30]), or using a composite measure of FA (e.g. [6]). Applying a Bonferroni correction would alter the significance of our result (required level of significance $<0.016$ versus $p$-value $=0.04$ ), and using the composite FA measure in our study did not show any relationship. Of course, some caution is also required when interpreting composite FAs because not all organs may show equivalent deviations in symmetry (see above) and, thus, composite scores may obscure some relationships. Thus, studies in other species and systems are needed before any general conclusions can be drawn.

The general lack of relationship between FA and immune response in the bush-cricket study populations could be an artifact of the timing of our measurements. Asymmetry results from some disruption to ideal growth during development [31], and once development is complete, these deviations to symmetry may persist for long periods during the remaining lifespan of the individual [10]. Thus, measures of FA in adult bush-crickets are likely to give some indication of environmental or individual quality during the development of these traits; however, the relationship between larval stress, adult FA and individual quality are complex and not easily predicted [32]. Similar complexities also plague immune response measurement, which is not fixed and may change during an individual's lifetime; constraints on immune response during development (i.e. the life-history period we are interested in regarding FA) may not be the same as those during adulthood (i.e. the life-history period we measured). For practical reasons we measured adult bush-crickets because they had completed their development (for FA measures) and they could be located for capture (only adult males stridulate). However, if trade-offs between optimal growth and immune response occur during development, but there is little or no correlation between immune response of developing individuals and adults, then correlations between FA and immune response may be difficult to find when sampling adults. It should also be remembered that by sampling adults we potentially introduce an additional bias. This occurs because we only sample individuals that have survived to adulthood; the interesting correlations between immune response and FA may be seen in those that tend to die during development leaving only the 'good' individuals, and, thus, little variation in FA / immune response correlations.

Relationships between FA, immune response and ecological variables present an exciting field of study and relate not only to evolutionary questions, but also questions from fields such as conservation biology and landscape ecology (e.g. (15, 33]). Unfortunately, relationships (or lack thereof) between these factors can be explained using current theory regardless of the direction of these relationships; for example, a negative correlation between immune defence and FA can be explained because poor environmental conditions act to increase FA while reducing immune function (summarised in [6]), while the opposite relationship can also be explained; immune defence and FA exhibit a positive relationship because immune defence is traded-off against some other attribute (e.g. symmetry; see [34] for an example of immune function being traded for sexual signalling). This is not intended as a general criticism, but rather to point out that the small number of empirical studies means it is difficult to validate or refine the underlying theory. There are many possible patterns and explanations for these patterns; however, we need many more studies from both lab and field-based populations before general patterns will start to emerge and the causes of these patterns can be properly elucidated. Now would be a good time to start investing in collecting these data. 


\section{Acknowledgements}

Thanks to Cecilia Erlandsson, David Levrén and Ingemar Lindqvist for help in the field and laboratory, and to two anonymous referees for their helpful comments on the manuscript.

\section{References}

1. Thornhill, R.; Møller, A.P. The relative importance of size and asymmetry in sexual selection. Behav. Ecol. 1998, 9, 546-551.

2. Field, S.A.; Yuval, B. Nutritional status effects copula duration in the Mediterranean fly, Ceratitis capitata (Insecta Tephritidae). Ethol. Ecol. Evol. 1999, 11, 61-70.

3. Parsons, P.A. Fluctuating asymmetry and stress intensity. Trends Ecol. Evol. 1990, 5, 97-98.

4. Schmid-Hempel, P. Variation in immune defence as a question of evolutionary ecology. Proc. $R$. Soc. B 2003, 270, 357-366.

5. Schmid-Hempel, R.; Schmid-Hempel, P. Colony performance and immunocompetence of a social insect, Bombus terrestris, in poor and variable environments. Funct. Ecol. 1998, 12, 22-30.

6. Rantala, M.J.; Ahtiainen, J.J.; Suhonen, J. Fluctuating asymmetry and immune function in a field cricket. Oikos 2004, 107, 479-484.

7. Rantala, M.J.; Roff, D.A. Inbreeding and extreme outbreeding cause sex differences in immune defence and life history traits in Epirrita autumnata. Heredity 2007, 98, 329-336.

8. Berggren, $\AA$. The effects of population and landscape ecology on body size in orthopterans. $J$. Orthopt. Res. 2008, 17, 183-188.

9. Schmid-Hempel, P. Evolutionary ecology of insect immune defences. Annu. Rev. Entomol. 2005, 50, 529-551.

10. Møller, A.P. A review of developmental instability, parasitism and disease infection, genetics and evolution. MEEGID 2006, 6, 133-140.

11. Civantos, E.; Ahnesjö, I.; Forsman, A. Immune function, parasitization and extended phenotypes in colour polymorphic pygmy grasshoppers. Biol. J. Linn. Soc. 2005, 85, 373-383.

12. Berggren, Å. Colonization success in Roesel's bush-cricket Metrioptera roeseli: the effects of propagule size. Ecology 2001, 82, 274-280.

13. Berggren, $\AA$. The effect of propagule size and landscape structure on morphological differentiation and asymmetry in experimentally introduced Roesel's bush-crickets. Conserv. Biol. 2005, 19, 1095-1102.

14. Berggren, Å.; Carlson, A.; Kindvall, O. The effect of landscape composition on colonization success, growth rate and dispersal in introduced bush-crickets Metrioptera roeseli. J. Anim. Ecol. 2001, 70, 663-670.

15. Berggren, $\AA$. Effects of population size, growth rate and landscape variables on immune responses in experimentally introduced populations of bush-crickets. Landscape Ecol. 2009, 24, 749-757.

16. Møller, A.P. 1996. Parasitism and developmental instability of hosts: a review. Oikos 1996, 77, 189-196.

17. Bellmann, H. Heuschrecken, beobachten, bestimmen; Verlag J. Neumann-Neudamm GmbH: Melsungen, Germany, 1985. 
18. Pettersson, B. Nya fyndplatser för cikadavårtbitare (Metrioptera roeseli) norr om Mälaren (New records of Roesel's bush-cricket, Metrioptera roeseli (Orthoptera: Tettigoniidae), north of Lake Mälaren, South-Central Sweden). Entomologisk Tidskrift 1996, 117, 121-122.

19. Marshall, J.A.; Haes, E.C.M. Grasshoppers and allied insects of Great Britain and Ireland; Harley Books: Martins, Great Horkesley, Colchester, Essex, UK, 1988.

20. Ingrisch, S. The plurennial life cycles of the European Tettigoniidae (Insecta: Orthoptera), 1. The effect of temperature in embryonic development and hatching. Oecologia 1986, 70, 606-616.

21. Allander, K.; Schmid-Hempel, P. Immune defence reaction in bumble-bee workers after a previous challenge and parasitic coinfection. Funct. Ecol. 2000, 14, 711-717.

22. Rantala, M.J.; Koskimäki, J.; Taskinen, J.; Tynkkynen, K.; Suhonen, J. Immunocompetence, developmental stability and wingspot size in the damselfly Calopteryx splendens L. Proc. R. Soc. B 2000, 267, 2453-2457.

23. Gillespie, J.P.; Kanost, M.R.; Trenczek, T. Biological mediators of insect immunity. Ann. Rev. Entomol. 1997, 42, 611-643.

24. Palmer, R.A.; Strobeck, C. Fluctuating asymmetry: measurement, analysis, patterns. Ann. Rev. Ecol. Syst. 1986, 17, 391-421.

25. Berggren, $\AA$. The effect of population and landscape ecology on body size in orthopterans. $J$. Orthoptera Res. 2008, 17, 183-188.

26. Gilmour, A.R.; Gogel, B.J.; Cullis, B.R.; Thompson, R. ASReml user guide release 2.0; VSN International Ltd: Hemel Hempstead, UK, 2006.

27. Vickery, V.R. Factors governing the distribution and dispersal of the recently introduced grasshopper, Metrioptera roeseli (Hgb.) (Orthoptera: Ensifera). Ann. Entomol. Soc. Québec 1965, 10, 165-171.

28. Gwynne, D.T. Katydids and bush-crickets: reproductive behaviour and evolution of the Tettigoniidae; Cornell University Press: USA, Ithaca, New York, 2001.

29. Rantala, M.J.; Kortet, R. Courtship song and immune function in the field cricket Gryllus bimaculatus. Biol. J. Linn. Soc. 2003, 79, 503-510.

30. Rice, W.R. Analyzing tables of statistical tests. Evolution 1989, 43, 223-225.

31. Møller, A.P. Developmental instability as a general measure of stress. Adv. Stud. Behav. 1998, 27, 181-213.

32. Campero, M.; De Block, M.; Ollevier, F.; Stoks, R. Metamorphosis offsets the link between larval stress. adult asymmetry and individual quality. Funct. Ecol. 2008, 22, 271-277.

33. French, S.S.; Fokidis, H.B. Variation in stress and innate immunity in the tree lizard (Urosaurus ornatus) across an urban-rural gradient. J. Comp. Physiol. B 2008, 178, 997-1005.

34. Ahtiainen, J.J.; Alatalo, R.V.; Kortet, R.; Rantala, M.J. A trade-off between sexual signalling and immune function in a natural population of the drumming wolf spider Hygrolycosa rubrofasciata. J. Evolution. Biol. 2005, 18, 985-991.

(C) 2009 by the authors; licensee Molecular Diversity Preservation International, Basel, Switzerland. This article is an open-access article distributed under the terms and conditions of the Creative Commons Attribution license (http://creativecommons.org/licenses/by/3.0/). 